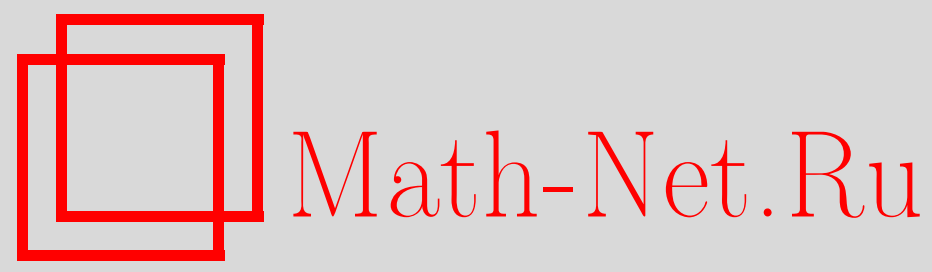

А. Л. Якымив, Допустимые функции для октанта, Матем. заметки, 2004, том 76, выпуск 3, 466-472

DOI: https://doi.org/10.4213/mzm113

Использование Общероссийского математического портала Math-Net.Ru подразумевает, что вы прочитали и согласны с пользовательским соглашением http://www . mathnet.ru/rus/agreement

Параметры загрузки:

IP : 35.173 .219 .12

26 апреля 2023 г., 16:45:33

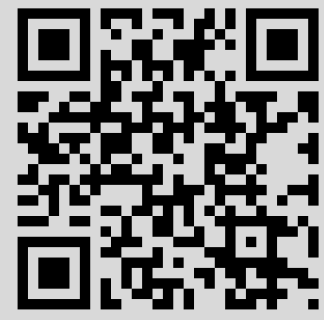




\title{
ДОПУСТИМЫЕ ФУНКЦИИ ДЛЯ ОКТАНТА
}

\section{А. Л. Якымив}

\begin{abstract}
В заметке приводятся примеры допустимых функций для октанта, являющихся многомерными обобщениями правильно меняющихся функций одной переменной, введенных в 1930 году Йованом Караматой. Допустимые функции для произвольного замкнутого выпуклого острого телесного $n$-мерного конуса были введены Ю. Н. Дрожжиновым и Б. И. Завьяловым в 1984 году в связи с приложениями в тауберовой теории и математической физике. Применения допустимых функций конуса при исследовании асимптотики многомерных безгранично делимых законов распределения на бесконечности получены в 2003 году автором.
\end{abstract}

Библиография: 18 названий.

1. Введение. Допустимые функции для произвольного замкнутого выпуклого острого телесного конуса в $\mathbb{R}^{n}$ были введены Дрожжиновым и Завьяловьм в 1984 году в работе [1] в связи с приложениями в тауберовой теории и математической физике. Они явились глубоким обобщением правильно меняющихся функций одной переменной, введенных в 1930 году Караматой [2], на многомерный случай.

В работе [3] автор использовал понятие допустимой функции при исследовании асимптотики на бесконечности многомерных безгранично делимых законов распределения, сосредоточенных в замкнутом выпуклом остром телесном однородном конусе.

Само по себе понятие допустимости трудно проверяемо непосредственно для большинства функций нескольких переменных. В связи с этим в работе Дрожжинова и Завьялова [4] и в книге Владимирова, Дрожжинова и Завьялова [5] был разобран ряд примеров таких функций.

В настоящей заметке мы продолжаем разбор примеров допустимых функций в случае, когда рассматриваемая область их определения является $n$-мерньм октантом $\mathbb{R}_{+}^{n}=$ $\left\{x: x=\left(x_{1}, \ldots, x_{n}\right) \in \mathbb{R}^{n}, x_{i}>0 \forall i=1, \ldots, n\right\}$.

2. Достаточные условия допустимости функций для октанта. Мы будем рассматривать произвольные семейства $U=\left\{U_{k}, k \in I \subseteq(0, \infty)\right\}$ линейных операторов, сохраняющих конус $\mathbb{R}_{+}^{n}$ инвариантньгм:

$$
U_{n} \mathbb{R}_{+}^{n}=\mathbb{R}_{+}^{n} \quad \forall k \in I,
$$

считая, что $\infty$ есть предельная точка $I$.

Работа вьполнена при финансовой поддержке Российского фонда фундаментальных исследований, грант № 02-01-01080, и программы президента РФ "Ведушие научные шшколы", грант № $\mathrm{H \amalg -}-1758.2003 .1$. 
ОПРЕДЕЛЕниЕ 1. Функция $g(x)$, определенная и положительная в $\mathbb{R}_{+}^{n}$, назьвается допустимой для $\mathbb{R}_{+}^{n}$, если для произвольного семейства линейных операторов $U=$ $\left\{U_{k}, k \in I \subseteq(0, \infty)\right\}$, сохраняющих инвариантньм $\mathbb{R}_{+}^{n}$, найдется подсемейство $V=$ $\left\{U_{k}, k \in J \subseteq I\right\}(\infty-$ предельная точка $J)$, для которого

1) существует вектор $x_{0} \in \mathbb{R}_{+}^{n}$ такой, что

$$
\frac{g\left(U_{k} x\right)}{g\left(U_{k} x_{0}\right)} \stackrel{x \in K}{\rightrightarrows} \varphi(x) \in(0, \infty), \quad k \rightarrow \infty, \quad k \in J
$$

где $K$ - произвольньй компакт из $\mathbb{R}_{+}^{n}$, а $\varphi(x)$ - непрерывная функция в $\mathbb{R}_{+}^{n}$;

2) справедлива оценка: существует $k_{0}$ такое, что

$$
\frac{g\left(U_{k} x\right)}{g\left(U_{k} x_{0}\right)} \leqslant \psi(x), \quad k>k_{0}, \quad k \in J, \quad x \in \mathbb{R}_{+}^{n},
$$

причем $\psi$ имеет медленньй рост в $\mathbb{R}_{+}^{n}$ :

$$
\int_{\mathbb{R}_{+}^{n}} \frac{\psi(x)}{1+|x|^{p}} d x<\infty
$$

для некоторого $p \geqslant 0$.

Пусть случайный вектор $\xi$ имеет безгранично делимое распределение в $\overline{\mathbb{R}}_{+}^{n}:$ в $\overline{\mathbb{R}}_{+}^{n}$ существует $\sigma$-конечная мера $\nu(d x)$ (возможно, неограниченная в окрестности нуля) такая, что

$$
\int_{|x| \leqslant 1}|x| \nu(d x)<\infty, \quad \int_{|x| \geqslant 1} \nu(d x)<\infty
$$

и

$$
\mathrm{M} e^{-(\lambda, \xi)}=\exp \left(\int_{\overline{\mathbb{R}}_{+}^{n}}\left(1-e^{-(\lambda, x)}\right) \nu(d x)\right)
$$

для произвольного $\lambda \in \overline{\mathbb{R}}_{+}^{n}$ (см. [6]). Мера $\nu$ назьвается спектральной мерой Леви безгранично делимого распределения случайного вектора $\xi$. Положим

$$
\widetilde{\mathbb{R}}_{+}^{n}(x)=\left\{y: y=\left(y_{1}, \ldots, y_{n}\right) \in \mathbb{R}_{+}^{n}, \exists i=1, \ldots, n: y_{i}>x_{i}\right\},
$$

где $x=\left(x_{1}, \ldots, x_{n}\right) \in \mathbb{R}_{+}^{n}$. В работе [3] показано, что если функция $g(x)=\nu\left(\widetilde{\mathbb{R}}_{+}^{n}(x)\right)$, $x \in \mathbb{R}_{+}^{n}$, является допустимой для $\mathbb{R}_{+}^{n}$, то

$$
\mathrm{P}\left\{\xi \in \widetilde{\mathbb{R}}_{+}^{n}(x)\right\}=(1+o(1)) g(x)
$$

при $x \in \mathbb{R}_{+}^{n}$ и $\min \left(x_{1}, \ldots, x_{n}\right) \rightarrow \infty$. Этот результат продолжает исследования асимптотики многомерных безгранично делимых распределений, проводившихся ранее в работах В. М. Круглова и его ученика С. Н. Антонова [7]-[15], М. С. Сгибнева [16], А. М. Улановского [17], Е. Омея [18] и автора [6]. В связи с упомянутьп результатом [3] представляет интерес разбор конкретных примеров допустимых функций $g(x)$ для $\mathbb{R}_{+}^{n}$. Займемся этим. 
ОПРЕДЕЛЕНИЕ 2. Функция $g(x)$, определенная, положительная, не возрастающая по каждой переменной и дифференцируемая на $\mathbb{R}_{+}^{n}$, называется сильно допустимой для $\mathbb{R}_{+}^{n}$, если для всех $i=1, \ldots, n$ и $x=\left(x_{1}, \ldots, x_{n}\right) \in \mathbb{R}_{+}^{n}$

$$
\frac{x_{i} \partial g(x) / \partial x_{i}}{g(x)} \geqslant \alpha>-1
$$

Если выполнено левое неравенство в (1) для некоторого конечного $\alpha$, то мы будем писать

$$
\text { ind } g=\min _{i=1, \ldots, n} \inf _{x \in \mathbb{R}_{+}^{n}} \frac{x_{i} \partial g(x) / \partial x_{i}}{g(x)} \text {. }
$$

В работе Дрожжинова и Завьялова [4] показано, что всякая сильно допустимая функция для $\mathbb{R}_{+}^{n}$ является допустимой функцией для $\mathbb{R}_{+}^{n}$ (см. также [5, гл. II, разд. 5.2, теорема 1]).

Справедлива следующая лемма.

ЛЕмма 1. Пусть функиии $f(x)$ и $g(x)$ сильно допустимы для $\mathbb{R}_{+}^{n}$. Тогда при $c>0$ функиии сf(x) и $f(x)+g(x)$ тожсе сильно допустимы для $\mathbb{R}_{+}^{n}$, причем

$$
\operatorname{ind}(c f)=\operatorname{ind} f
$$

$u$

$$
\operatorname{ind}(f+g) \geqslant \min (\text { ind } f, \text { ind } g) \text {. }
$$

ДокАЗАТЕЛЬСтво. Утверждение леммы 1 относительно $c f(x)$ следует сразу из определения сильной допустимости функции. Докажем утверждение леммы 1 относительно $f(x)+g(x)$. Так как $f(x)$ и $g(x)$ не возрастают в $\mathbb{R}_{+}^{n}$, то ind $f \leqslant 0$ и ind $g \leqslant 0$, поэтому для произвольных $i=1, \ldots, n$ и $x \in \mathbb{R}_{+}^{n}$

$$
\begin{aligned}
\frac{x_{i} \partial f(x) / \partial x_{i}+x_{i} \partial g(x) / \partial x_{i}}{f(x)+g(x)} & \geqslant \frac{f(x) \text { ind } f+g(x) \text { ind } g}{f(x)+g(x)} \\
& \geqslant \frac{f(x) \min (\text { ind } f, \text { ind } g)+g(x) \min (\text { ind } f, \text { ind } g)}{f(x)+g(x)} \\
& =\min (\text { ind } f, \text { ind } g)>-1,
\end{aligned}
$$

откуда следует (4). Лемма 1 доказана.

Через $\mathfrak{F}$ обозначим класс функций распределения на $\mathbb{R}_{+}^{1}$ таких, что для произвольной функции распределения $F(x) \in \mathfrak{F}$ верно представление

$$
1-F(x) \equiv \bar{F}(x)=\exp \left(-\int_{0}^{x} \frac{\varepsilon(u)}{u} d u\right) \quad \forall x \geqslant 0
$$


для некоторой измеримой функции $\varepsilon(x)$ на $\mathbb{R}_{+}^{1}$, обладающей свойствами:

$$
\begin{gathered}
0 \leqslant \inf _{0 \leqslant x<\infty} \varepsilon(x) \leqslant \sup _{0 \leqslant x<\infty} \varepsilon(x)<1, \\
\int_{0}^{x} \frac{\varepsilon(u)}{u} d u<\infty \quad \text { для произвольного } x \in(0, \infty), \\
\int_{0}^{\infty} \frac{\varepsilon(u)}{u} d u=\infty
\end{gathered}
$$

Заметим, что произвольная измеримая функция $\varepsilon(x)$, обладающая свойствами $(6)-(8)$, определяет некоторую функцию распределения $F(x)$, удовлетворяющую (5). В самом деле, из (6) следует, что $\varepsilon(x)$ неотрицательна при $x \geqslant 0$, стало быть, $\bar{F}(x)$ невозрастает, а $F(x)$ не убывает при $x \geqslant 0$. Кроме того, из (5) следует, что $F(0)=0$, а из (8) следует, что $F(\infty)=1$. Таким образом, условия (6)-(8) действительно определяют некоторую функцию распределения $F(x)$.

ТЕорема 1. Пусть спектральная мера Леви $\nu$ безгранично делимого распределения абсолютно непрерывна на $\mathbb{R}_{+}^{n}$ и имеет вид

$$
\nu(A)=\int_{A} h(x) d x
$$

где $A$ - произвольное борелевское множество из $\mathbb{R}_{+}^{n}$,

$$
h(x)=\sum_{i=1}^{m} c_{i} h_{i}(x), \quad x \in \mathbb{R}_{+}^{n}
$$

а $c_{i}>0, i=1, \ldots, m,-$ некоторые полохительные постоянные,

$$
h_{i}(x)=h_{i 1}\left(x_{1}\right) \cdots h_{i n}\left(x_{n}\right)
$$

для произвольных $x=\left(x_{1}, \ldots, x_{n}\right) \in \mathbb{R}_{+}^{n} u i=1, \ldots m$, где $h_{i j}(t)-$ плотности функиий распределения $F_{i j}(t) \in \mathfrak{F}$ для всех $i=1, \ldots, m u j=1, \ldots, n$. Тогда функиия $g(x)=\nu\left(\widetilde{\mathbb{R}}_{+}^{n}(x)\right), x \in \mathbb{R}_{+}^{n}$, сильно допустима для конуса $\mathbb{R}_{+}^{n}$.

ДокАЗАТЕЛЬСТво. В силу леммы 1 достаточно проверить справедливость теоремы 1 для случая $m=1$ и $c_{1}=1$. Тогда при $x=\left(x_{1} \ldots, x_{n}\right) \in \mathbb{R}_{+}^{n}$

$$
h(x)=h_{1}\left(x_{1}\right) \cdots h_{n}\left(x_{n}\right),
$$


где $h_{i}(t)$ - плотности функций распределения $F_{i}(t) \in \mathfrak{F}, i=1, \ldots, n$. Мы имеем при $x=\left(x_{1}, \ldots, x_{n}\right) \in \mathbb{R}_{+}^{n}$ и $\mathbb{R}_{+}^{n}(x)=\left\{y: y=\left(y_{1}, \ldots, y_{n}\right) \in \mathbb{R}_{+}^{n}, y_{i} \leqslant x_{i} \forall i=1, \ldots, n\right\}$

$$
\begin{aligned}
0 & \geqslant \frac{x_{1} \partial g(x) / \partial x_{1}}{g(x)} \\
& =\frac{x_{1} \frac{\partial}{\partial x_{1}}\left(\nu\left(\mathbb{R}_{+}^{n}\right)-\int_{\mathbb{R}_{+}^{n}(x)} h_{1}\left(y_{1}\right) \cdots h_{n}\left(y_{n}\right) d y\right)}{\nu\left(\mathbb{R}_{+}^{n}\right)-\int_{\mathbb{R}_{+}^{n}(x)} h_{1}\left(y_{1}\right) \cdots h_{n}\left(y_{n}\right) d y} \\
& =\frac{x_{1} \frac{\partial}{\partial x_{1}}\left(1-F_{1}\left(x_{1}\right) \cdots F_{n}\left(x_{n}\right)\right)}{1-F_{1}\left(x_{1}\right) \cdots F_{n}\left(x_{n}\right)}=\frac{-x_{1} h_{1}\left(x_{1}\right) F_{2}\left(x_{2}\right) \cdots F_{n}\left(x_{n}\right)}{1-F_{1}\left(x_{1}\right) \cdots F_{n}\left(x_{n}\right)} \\
& \geqslant \frac{-x_{1} h_{1}\left(x_{1}\right) F_{2}\left(x_{2}\right) \cdots F_{n}\left(x_{n}\right)}{F_{2}\left(x_{2}\right) \cdots F_{n}\left(x_{n}\right)-F_{1}\left(x_{1}\right) \cdots F_{n}\left(x_{n}\right)} \\
& =-\frac{x_{1} h_{1}\left(x_{1}\right)}{\bar{F}_{1}\left(x_{1}\right)}=-\varepsilon_{1}\left(x_{1}\right) \geqslant-\alpha_{1}>-1,
\end{aligned}
$$

где $\varepsilon_{1}(t)$ - функция из представления (5), соответствующая функции распределения $F_{1}(t)$,

$$
\alpha_{1}=\sup _{0 \leqslant t<\infty} \varepsilon_{1}(t)<1
$$

Точно так же для некоторых чисел $\alpha_{i} \in(0,1)$ и всех $i=1, \ldots, n$ мы имеем при $x=\left(x_{1}\right.$, $\left.\ldots, x_{n}\right) \in \mathbb{R}_{+}^{n}$

$$
0 \geqslant \frac{x_{i} \partial g(x) / \partial x_{i}}{g(x)} \geqslant-\alpha_{i}>-1
$$

Теорема 1 доказана.

Теорема 2. Пусть заданы некоторые числа $p, q \in \mathbb{N}$, постоянные $c_{j}>0, \beta_{j} \in \mathbb{R}^{1}$, векторы $l_{j} \in \mathbb{R}_{+}^{n}, \alpha_{j} \in \mathbb{R}^{n}, j=1, \ldots, p+q$, и возрастающая положсительная дифферениируемая функиия $r(t), t \in \mathbb{R}_{+}^{1}$, причем

$$
\frac{t \partial r(t) / \partial t}{r(t)} \leqslant b<\infty \quad \forall t \in \mathbb{R}_{+}^{1}
$$

$u$

$$
\begin{aligned}
& \alpha_{i j} \beta_{j}<0, \quad i=1, \ldots, n, \quad j=1, \ldots, p, \\
& \alpha_{i j} \beta_{j}>0, \quad i=1, \ldots, n, \quad j=p+1, \ldots, p+q \text {. }
\end{aligned}
$$

Eсли

$$
\gamma=\left(\min _{i=1, \ldots, n} \min _{j=1, \ldots, p} \alpha_{i j} \beta_{j}-\max _{i=1, \ldots, n j=p+1, \ldots, p+q} \max _{i j} \beta_{j}\right) b>-1,
$$

то функиия

$$
g(x)=r\left(\frac{\sum_{j=1}^{p} c_{j}\left(l_{j}, x^{\alpha_{j}}\right)^{\beta_{j}}}{\sum_{j=p+1}^{p+q} c_{j}\left(l_{j}, x^{\alpha_{j}}\right)^{\beta_{j}}}\right), \quad x \in \mathbb{R}_{+}^{n},
$$

сильно допустима для $\mathbb{R}_{+}^{n}$. Здесь

$$
x^{\alpha_{j}}=\left(x_{1}^{\alpha_{1 j}}, \ldots, x_{n}^{\alpha_{n j}}\right) .
$$

Настоящая теорема обобщает соответствующую теорему из книги Владимирова, Дрожжинова и Завьялова [5, гл. II, разд. 5.4] на случай произвольных степеней $\alpha_{j}$ вектора $x$ (там были $\left.\alpha_{j} \equiv(1,1, \ldots, 1), j=1, \ldots, p+q\right)$. 
ДокАЗАТЕЛЬСТво ТЕОРЕмЫ 2. При $l \in \mathbb{R}_{+}^{n}, \alpha \in \mathbb{R}^{n}, \beta \in \mathbb{R}^{1}, \alpha_{i} \beta<0, i=1, \ldots, n$, для произвольных $x \in \mathbb{R}_{+}^{n}$ мы имеем

$$
\frac{x_{i} \frac{\partial}{\partial x_{i}}\left(l, x^{\alpha}\right)^{\beta}}{\left(l, x^{\alpha}\right)^{\beta}}=\frac{x_{i} \alpha_{i} l_{i} x_{i}^{\alpha_{i}-1} \beta_{i}\left(l, x^{\alpha}\right)^{\beta-1}}{\left(l, x^{\alpha}\right)^{\beta}}=\frac{\alpha_{i} \beta l_{i} x_{i}^{\alpha_{i}}}{\left(l, x^{\alpha}\right)} \geqslant \alpha_{i} \beta .
$$

Поэтому, пользуясь соотношением (13), точно так же, как и в лемме 1 , мы устанавливаем, что при

$$
u(x)=\sum_{j=1}^{p} c_{j}\left(l_{j}, x^{\alpha_{j}}\right)^{\beta_{j}}
$$

и

$$
v(x)=\sum_{j=p+1}^{p+q} c_{j}\left(l_{j}, x^{\alpha_{j}}\right)^{\beta_{j}}
$$

для произвольных $k=1, \ldots, n, x \in \mathbb{R}_{+}^{n}$ имеет место неравенство

$$
\frac{x_{k} \frac{\partial u(x)}{\partial x_{k}}}{u(x)} \geqslant \min _{i=1, \ldots, n} \min _{j=1, \ldots, p} \alpha_{i j} \beta_{j}
$$

и неравенство

$$
\frac{x_{k} \frac{\partial v(x)}{\partial x_{k}}}{v(x)} \leqslant \max _{i=1, \ldots, n j=p+1, \ldots, p+q} \max _{i j} \beta_{j} .
$$

Полагая $w(x)=u(x) / v(x), x \in \mathbb{R}_{+}^{n}$, мы имеем при $k=1, \ldots, n, x \in \mathbb{R}_{+}^{n}$

$$
\begin{aligned}
\frac{x_{k} \frac{\partial w(x)}{\partial x_{k}}}{w(x)} & =\frac{x_{k} \frac{\partial u(x)}{\partial x_{k}} v(x)}{u(x) v(x)}-\frac{x_{k} \frac{\partial v(x)}{\partial x_{k}} u(x) v(x)}{u(x) v^{2}(x)}=\frac{x_{k} \frac{\partial u(x)}{\partial x_{k}}}{u(x)}-\frac{x_{k} \frac{\partial v(x)}{\partial x_{k}}}{v(x)} \\
& \geqslant \min _{i=1, \ldots, n} \min _{j=1, \ldots, p} \alpha_{i j} \beta_{j}-\max _{j=p+1, \ldots, p+q} \alpha_{i j} \beta_{j} .
\end{aligned}
$$

Поскольку $g(x)=r(w(x))$, то при $k=1, \ldots, n$ и $x \in \mathbb{R}_{+}^{n}$ в силу соотношений $(12)$ и $(16)$ имеем

$$
\begin{aligned}
\frac{x_{k} \frac{\partial g(x)}{\partial x_{k}}}{g(x)} & =\frac{x_{k} r^{\prime}(w(x)) \frac{\partial w(x)}{\partial x_{k}}}{r(w(x))}=\frac{x_{k} r^{\prime}(w(x)) \frac{\partial w(x)}{\partial x_{k}} w(x)}{r(w(x)) w(x)} \\
& \geqslant\left(\min _{i=1, \ldots, n j=1, \ldots, p} \min _{i j} \beta_{j}-\max _{i=1, \ldots, n j=p+1, \ldots, p+q} \alpha_{i j} \beta_{j}\right) b>-1
\end{aligned}
$$

согласно (12). Теорема 2 доказана.

Другие примеры сильно допустимых функций для $\mathbb{R}_{+}^{n}$ можно строить аналогичньм образом, в частности, образуя линейные комбинации функций из теоремы 1 и теоремы 2 , рассматривая произведения такого рода функций (в последнем случае дополнительно нужно следить за выполнением правого неравенства в соотношении (1)). 


\section{СПИСОК ЦИТИРОВАННОЙ ЛИТЕРАТУРЫ}

[1] Дрожжинов Ю. Н., Завьялов Б. И. Теоремы сравнения тауберова типа // Докл. АН CCCP. 1984. T. 279. № 3. С. 532-535.

[2] Karamata J. Sur un mode croissante régulière des fonctions // Mathematica. 1930. V. 4. P. 38-53.

[3] Якымив А. Л. Тауберовы теоремы и асимптотика безгранично делимых распределений в конусе // Теория вероятн. и ее примен. 2003. Т. 48. №3. С. 487-502.

[4] Дрожжинов Ю.Н., Завьялов Б. И. Многомерные тауберовы теоремы сравнения для обобщенных функций в конусах // Матем. сб. 1985. Т. 126(168). №4. С. 515-542.

[5] Владимиров В.С., Дрожжинов Ю.Н., Завьялов Б.И. Многомерные тауберовы теоремы для обобщенных функций. М.: Наука, 1986.

[6] Yakymiv A. L. The asymptotics of multidimesional infinitely divisible distributions // J. Math. Sci. 1997. V. 84. № 3. P. 1197-1207.

[7] Антонов С. Н. К асимптотическому поведению безгранично делимых законов // Матем. заметки. 1980. Т. 28. №6. С. 939-946.

[8] Антонов С. Н., Круглов В. М. Об асимптотическом поведении безгранично делимых распределений в банаховом пространстве // Теория вероятн. и ее примен. 1982. Т. 27. № 4 . C. $625-642$.

[9] Антонов С.Н., Круглов В. М. Еще раз об асимптотическом поведении безгранично делимых распределений в банаховом пространстве // Теория вероятн. и ее примен. 1984. T. 29. № 4. C. $735-742$.

[10] Круглов В. М. Замечание к теории безгранично делимых законов // Теория вероятн. и ее примен. 1970. Т. 15. № 2. С. 330-336.

[11] Круглов В. М. Интегралы по безгранично делимым распределениям в гильбертовом пространстве // Матем. заметки. 1972. Т. 11. №6. С. 669-676.

[12] Круглов В. М. Сходимость числовых характеристик сумм независимых случайных величин со значениями в гильбертовом пространстве // Теория вероятн. и ее примен. 1973. Т. 18. № 4. C. $734-752$.

[13] Круглов В. М. О безгранично делимых распределениях в гильбертовом пространстве // Матем. заметки. 1974. Т. 16. № 4. С. 585-594.

[14] Круглов В. М. Характеризация одного класса распределений в гильбертовом пространстве // Матем. заметки. 1974. Т. 16. № 5. С. 777-782.

[15] Круглов В. М. Дополнительные главы теории вероятностей. М.: Высшая школа, 1984.

[16] Сгибнев М. С. Асимптотика безгранично делимых распределений в $\mathbb{R}^{n} / /$ Tp. ин-та матем. CO АH CCCP. 1989. T. 13. C. 100-116.

[17] Улановский А. М. Об асимптотическом поведении безгранично делимых распределений на бесконечности // Теория функций, функцион. анализ и их прилож. 1981. Т. 35. С. 100-107.

[18] Omey E. Infinite divisibility and random sums of random vectors // Yokohama Math. J. 1985. V. 33. №1-2. P. 39-48.

Математический институт РАН им. В.А. Стеклова

Поступило

E-mail: arsen@mi.ras.ru

28.10 .2003 